\title{
Circulating prolactin level is increased in metabolically healthy obesity
}

\author{
Jia Liu, Lin Zhang, Jing Fu, Qiu Wang and Guang Wang \\ Department of Endocrinology, Beijing Chao-yang Hospital, Capital Medical University, Chaoyang District, Beijing, China
}

Correspondence should be addressed to G Wang: drwg6688@126.com

\begin{abstract}
Objective: Prolactin (PRL) has been demonstrated as a metabolic hormone to regulate energy metabolism recently. The present study aims to investigate the association between PRL and metabolic alterations in different obesity phenotypes.

Methods: A total of 451 drug-naive participants were recruited, comprising 351 obese patients and 100 age- and sex-matched healthy participants with normal weight. PRL, anthropometric, and clinical parameters were measured.

Results: In the obesity group, 15.1\% (53/351) were categorized as 'metabolically healthy obesity (MHO)'. Besides favorable blood pressure, glucose, and lipids profiles, the $\mathrm{MHO}$ group exhibited increased PRL, and lower levels of high-sensitivity C-reactive protein (hsCRP), homeostasis model assessment of insulin resistance (HOMA-IR), and adipose tissue insulin resistance (adipo-IR) than the metabolically unhealthy obesity (MUHO) group (PRL, HOMA-IR, and adipo-IR: $P<0.01$; hsCRP: $P<0.05$ ). The severe MUHO group showed significantly decreased PRL levels than the mild MUHO group $(P<0.05)$. Multivariate linear regression analysis indicated that fasting plasma glucose (FBG) and adipo-IR were significantly associated with PRL (FBG: $\beta=-0.263, P<0.05$; adipo-IR: $\beta=-0.464, P<0.01)$. Multivariable logistic regression analysis showed that hsCRP $(\mathrm{OR}=0.824)$ and $\mathrm{PRL}(\mathrm{OR}=1.211)$ were independent predictors of $\mathrm{MHO}$ (all $P<0.01)$. Conclusion: The MHO group had significantly increased circulating PRL levels when compared with the control and MUHO groups, and multivariable logistic regression analysis showed that PRL was independent predictors of $\mathrm{MHO}$. Our findings suggested that increased circulating PRL might be a compensatory response for favoring energy metabolism during obesity.
\end{abstract}

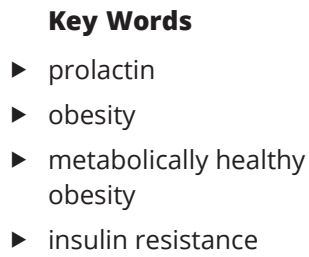

Endocrine Connections (2021) 10, 484-491

\section{Introduction}

The global epidemic of obesity remains a growing public health concern owing to its short- and long-term adverse health sequelae $(1,2)$. Epidemiological studies have demonstrated that obesity is associated with increased risk of hypertension, dyslipidemia, and type 2 diabetes, further resulting in the development of cardiovascular diseases (2, 3 ). However, recent studies have found that a proportion of obese subjects appear to have a favorable metabolic profile with no aforementioned metabolic abnormalities, which was called 'metabolically healthy obesity (MHO)'
$(4,5,6,7)$. The precise mechanisms responsible for such a favorable metabolic phenotype in obesity are not entirely understood.

Prolactin (PRL) is secreted from anterior pituitary and named for its crucial effect on lactation $(8,9)$. Recent studies have demonstrated that PRL plays an important role in regulating energy metabolism $(8,9,10,11,12$, $13,14,15,16)$. PRL administration increased insulin sensitivity and regulated glucose and lipid metabolism in rodent models $(10,11,12)$. PRL receptor knockout caused
This work is licensed under a Creative Commons Attribution-NonCommercial 4.0 International License. ded from Bioscientifica.com at 04/26/2023 10:04:40AM 
insulin resistance and severe adipose tissue dysfunction in obese mice (10). Many population-based studies found that a relatively higher PRL level was associated with lower risk of diabetes, nonalcoholic fatty liver disease and metabolic syndrome $(12,13,14,15)$. The previous study showed that obese patients had different PRL levels compared to nonobese participants $(15,16,17)$. As an intermediate stage from healthy to overt metabolic abnormalities, MHO patients may have different PRL levels $(7,18)$. However, until now, few reports regarding the correlation of PRL and $\mathrm{MHO}$ have been published. The present study aims to investigate the association between PRL and metabolic alterations in different obesity phenotypes.

\section{Materials and methods}

\section{Study design and participants}

This study consecutively recruited 351 obese patients $\left(\mathrm{BMI} \geq 30.0 \mathrm{~kg} / \mathrm{m}^{2}\right)$ attending the Obesity Clinic at Beijing Chao-yang Hospital Affiliated to Capital Medical University from January 2019 to December 2019 (2). Meanwhile, 100 age- and sex-matched healthy controls with normal weight $\left(18.5 \mathrm{~kg} / \mathrm{m}^{2} \leq \mathrm{BMI}<25.0 \mathrm{~kg} / \mathrm{m}^{2}\right)$ were consecutively enrolled from the Physical Examination Center at the same hospital (2). All participants were between 18 and 50 years old. No participants had a history of smoking (in the last 6 months), alcohol abuse, cardiovascular disease, thyroid dysfunction, disorders in thalamus and pituitary, polycystic ovarian syndrome, hypogonadism, severe hepatic insufficiency, renal function impairment, acute or chronic infections, systemic inflammatory disease, or cancer. Participants who were pregnant, lactating, or took any medications which influence PRL, glucose, lipid, or blood pressure were excluded. In order to investigate the association between PRL and metabolic alterations in the early stages of metabolic abnormalities, participants with a history of diabetes were excluded from the present study. We also excluded participants with PRL $\geq$ three times the upper limit of normal (ULN) or with oligo-/ amenorrhoea or/and -galactorrhoea $(19,20)$. Polyethylene glycol precipitation was routinely taken for patients with hyperprolactinemia in order to exclude the possibility of suffering from macroprolactinemia. This study was conducted in accordance with the Declaration of Helsinki ethical principles. The protocol was approved by the Ethics Committee of Beijing Chao-yang Hospital affiliated with Capital Medical University. All enrolled participants provided a written informed consent.

\section{Measurements of clinical parameters}

A standard questionnaire was performed to collect information about health status and medications. Anthropometric measurements were conducted after overnight fasting. Alcohol consumption and intercourse were forbidden $24 \mathrm{~h}$ before the examination. Height and body weight were respectively measured to the nearest $0.1 \mathrm{~cm}$ and $0.1 \mathrm{~kg}$, with participants wearing indoor clothes and no shoes, by the same trained group. Waist circumference (WC) and hip circumference (HC) were measured to the nearest $0.1 \mathrm{~cm}$ between the lower rib margin and the iliac crest and at the maximum width of the buttocks in the horizontal plane, using an anthropometric tape, with the patient standing straight, abdomen relaxed, and feet together. Blood pressure was measured twice after a 5-min rest in a supine position, and systolic blood pressure (SBP) and diastolic blood pressure (DBP) were calculated as the average of two measurements. BMI was calculated as weight divided by height squared $\left(\mathrm{kg} / \mathrm{m}^{2}\right)$.

Venous blood samples were obtained between 08:00 and 09:00 h. after overnight fasting. The serum levels of total cholesterol (TC), triglyceride (TG), high-density lipoprotein cholesterol (HDL-C), low-density lipoprotein cholesterol (LDL-C) and non-esterified fatty acid (NEFA) were measured by colorimetric enzymatic assays using an autoanalyzer (Hitachi 747, Roche Diagnostics). Fasting plasma glucose (FBG) was measured by glucose oxidase method (Hitachi 747, Roche Diagnostics). Fasting plasma insulin (FINS) was estimated by the chemiluminescence method (Dimension Vista, Siemens Healthcare Diagnostics). Hemoglobin A1c (HbA1c) was measured by high-performance liquid chromatography, using an HLC-723G7 analyzer (Tosoh Corporation, Tokyo, Japan). High-sensitivity C-reactive protein (hsCRP) was measured using an immunonephelometric assay. Estradiol (normal range: $<56.0 \mathrm{pg} / \mathrm{mL}$ for male), total testosterone (TT) (normal range: $1.60-7.26 \mathrm{ng} / \mathrm{mL}$ for male; $<0.73 \mathrm{ng} / \mathrm{mL}$ for female), thyroid stimulating hormone (TSH) (normal range: $0.55-4.78 \mu \mathrm{IU} / \mathrm{mL}$ ), and PRL (normal range: $2.5-17.0 \mathrm{ng} / \mathrm{mL}$ for male; $1.9-25.0 \mathrm{ng} / \mathrm{mL}$ for female) were measured using a chemiluminescent immunometric assay by the Immulite 1000 Immunoassay Analyzer (Siemens). Homeostasis model assessment of insulin resistance (HOMA-IR) was calculated according to the following formulas: HOMA-IR $=$ FINS $(\mu \mathrm{IU} / \mathrm{mL}) \times \mathrm{FBG}(\mathrm{mmol} / \mathrm{L}) / 22.5$ (21). Adipose tissue insulin resistance (adipo-IR) was calculated according to the formula: Adipo-IR=fasting NEFA $(\mathrm{mmol} / \mathrm{L}) \times$ FINS $(\mathrm{pmol} / \mathrm{L})(22)$.

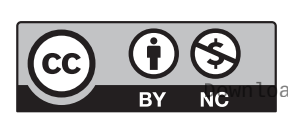

This work is licensed under a Creative Commons Attribution-NonCommercial 4.0 International License. ded from Bioscientifica.com at 04/26/2023 10:04:40AM 


\section{Definition of $\mathrm{MHO}$}

The present study used a strict definition of MHO proposed by Ortega (23). An individual was categorized as MHO if BMI $\geq 30 \mathrm{~kg} / \mathrm{m}^{2}$ and met none of the following criteria: (i) elevated TG ( $\geq 1.7 \mathrm{mmol} / \mathrm{L})$, (ii) reduced HDL-C $(<1.0$ $\mathrm{mmol} / \mathrm{L}$ for men and $<1.3 \mathrm{mmol} / \mathrm{L}$ for women), (iii) elevated SBP $(\geq 130 \mathrm{mmHg})$ or/and DBP $(\geq 85 \mathrm{mmHg})$, and (iv) elevated FBG ( $\geq 5.6 \mathrm{mmol} / \mathrm{L})$. Obese patients with one or more of the four metabolic risk components were categorized as metabolically unhealthy obesity (MUHO) (23).

\section{Statistical analysis}

Data were analyzed using SPSS 22.0 (SPSS). The Kolmogorov-Smirnov test was used to evaluate the distribution of continuous data. Normally distributed variables were expressed as mean \pm s.D., while variables with a skewed distribution, including TG, FINS, hsCRP, HOMA-IR, adipo-IR, estradiol, and TT, were given as the median, upper and lower quartiles. The differences in continuous data between the three groups (control, $\mathrm{MHO}$, and MUHO groups) were analyzed using ANOVA or Kruskal-Wallis $\mathrm{H}$ test followed by a post hoc test. The differences between two groups (two subgroups in the MUHO group) were analyzed using an independent $t$-test for normally distributed variables or a Mann-Whitney $U$-test for skewed-distribution variables. The proportions were analyzed using chi-squared tests. Pearson or Spearman correlation was used to test the correlation between two parameters. Multivariate linear regression analysis was performed to assess the relationship between PRL and metabolic parameters. Multivariable logistic regression analysis was performed to find the independent predictors for $\mathrm{MHO}$ in obese people. Statistical significance was considered at $P<0.05$ (two-tailed).

\section{Results}

\section{Clinical characteristics of control, MHO, and MUHO groups}

A total of 451 participants comprising 351 obese patients and 100 age- and sex-matched healthy controls were included in the present study. The clinical characteristics of the control, $\mathrm{MHO}$, and MUHO groups were summarized in Table 1. Among 351 obese patients, 15.1\% (53/351) were categorized as $\mathrm{MHO}$. The control, $\mathrm{MHO}$ and MUHO groups were comparable with respect to age and gender. Among the three groups, there were no significant differences in estradiol and TT levels. Significant differences were observed for BMI, WC, HC, SBP, DBP, TC, TG, HDL-C, LDL-C, NEFA, FBG, FINS, HbA1c, hsCRP, HOMA-IR, adipo-IR, TSH, and PRL levels among three groups (BMI, WC, HC, SBP, DBP, TC, TG, HDL-C, FINS, HbA1c, HOMA-IR, and adipo-IR: $P<0.01$; LDL-C, NEFA, FBG, hsCRP, and TSH: $P<0.05)$.

We next performed post hoc analyses and found that both $\mathrm{MHO}$ and MUHO groups had significantly increased BMI, WC, HC, TC, TG, FINS, hsCRP, HOMA-IR, and adipo-IR levels when compared with the control group (Table 1). The significant differences in SBP, DBP, HDL-C, LDL-C, NEFA, FBG, HbA1c, and TSH were observed when comparing the MUHO group with the control group (Table 1). The MHO group had decreased SBP, DBP, FBG, HbA1c, hsCRP, HOMA-IR, and adipo-IR levels and increased HDL-C levels, as compared to the MUHO group (Table 1). Interestingly, the MHO group had significantly higher PRL levels than the control and MUHO groups, while no significant difference in PRL level was observed in the latter two groups (Fig. 1 and Table 1).

\section{Clinical characteristics in MUHO subjects with different degrees of metabolic abnormality}

The MUHO patients were further divide into the mild MUHO group (MUHO patients with one or two metabolic risk components) and severe MUHO group (MUHO patients with three or four metabolic risk components) according to the number of the following metabolic riskcomponents: (i) elevated TG ( $\geq 1.7 \mathrm{mmol} / \mathrm{L})$, (ii) reduced HDL-C $(<1.0 \mathrm{mmol} / \mathrm{L}$ for men and $<1.3 \mathrm{mmol} / \mathrm{L}$ for women), (iii) elevated SBP ( $\geq 130 \mathrm{mmHg}$ ) or/and DBP ( $\geq 85 \mathrm{mmHg}$ ), and (iv) elevated FBG ( $\geq 5.6 \mathrm{mmol} / \mathrm{L}$ ). There were no significant differences in age, gender, BMI, WC, HC, TC, LDL-C, HbA1c, estradiol, TT, or TSH between mild and severe MUHO groups (all $P>0.05$, Table 2 ). The severe MUHO group had significantly higher levels of SBP, DBP, TG, NEFA, FBG, FINS, hsCRP, HOMA-IR, and adipo-IR, and lower levels of HDL-C and PRL when compared with the mild MUHO group (SBP, DBP, TG, HDL-C, FBG, and HOMA-IR: $P<0.01$; NEFA, FINS, hsCRP, adipo-IR, and PRL: $P<0.05$; Table 2).

\section{The correlations between PRL and clinical parameters in all participants}

Bivariate correlation analyses were used to investigate the correlations between circulating PRL levels and clinical parameters in all participants. The circulating PRL levels were

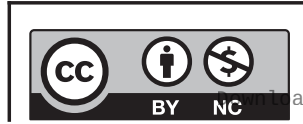

This work is licensed under a Creative Commons Attribution-NonCommercial 4.0 International License. ded from Bioscientifica.com at 04/26/2023 10:04:40AM via free access 
Table 1 Clinical characteristics of control, MHO, and MUHO groups.

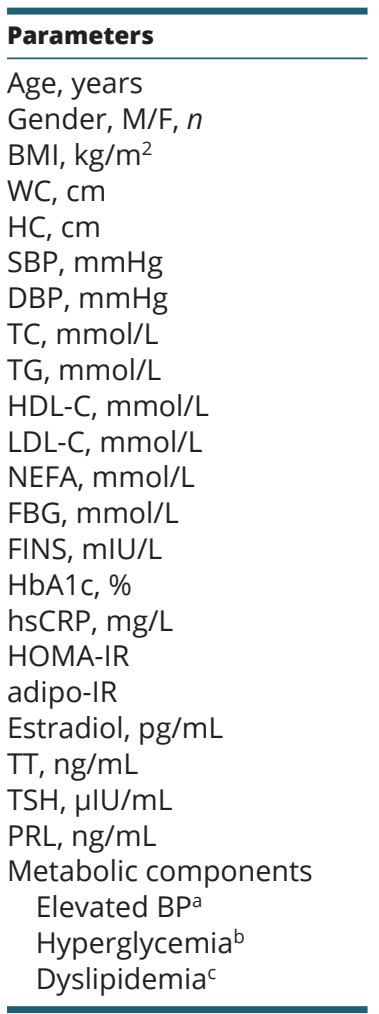

\begin{tabular}{c}
\hline Control group $(n=100)$ \\
\hline $32.3 \pm 6.7$ \\
$35 / 65$ \\
$21.43 \pm 2.68$ \\
$79.92 \pm 6.57$ \\
$89.61 \pm 6.10$ \\
$119.3 \pm 5.2$ \\
$70.2 \pm 4.9$ \\
$4.57 \pm 0.90$ \\
$0.89(0.70-1.27)$ \\
$1.49 \pm 0.34$ \\
$2.69 \pm 0.81$ \\
$0.52 \pm 0.34$ \\
$4.35 \pm 0.45$ \\
$5.46(2.01-10.04)$ \\
$5.20 \pm 0.29$ \\
$0.22(0.06-0.70)$ \\
$1.43(0.63-3.05)$ \\
$26.37(5.64-44.29)$ \\
$50.60(32.08-84.42)$ \\
$2.19(0.34-4.03)$ \\
$2.26 \pm 1.10$ \\
$12.40 \pm 7.12$
\end{tabular}

\begin{tabular}{c}
\hline MHO group $(n=53)$ \\
\hline $32.2 \pm 7.6$ \\
$19 / 34$ \\
$34.09 \pm 2.79 * *$ \\
$106.81 \pm 11.39 * *$ \\
$117.00 \pm 4.12 *$ \\
$121.9 \pm 5.7$ \\
$74.6 \pm 7.3$ \\
$4.93 \pm 0.69 *$ \\
$1.25(0.89-1.49)^{*}$ \\
$1.32 \pm 0.18$ \\
$2.90 \pm 0.52$ \\
$0.60 \pm 0.22$ \\
$4.39 \pm 0.70$ \\
$18.80(10.07-26.09)^{* *}$ \\
$5.30 \pm 0.35$ \\
$2.87(0.80-4.97)^{* *}$ \\
$3.30(1.89-5.68)^{*}$ \\
$57.85(23.22-95.99)^{*}$ \\
$93.00(47.00-179.51)$ \\
$2.90(0.88-4.77)$ \\
$2.47 \pm 1.20$ \\
$19.19 \pm 5.59 * *$
\end{tabular}

\begin{tabular}{c}
\hline MUHO group $(n=298)$ \\
\hline $31.5 \pm 7.2$ \\
$101 / 197$ \\
$34.00 \pm 2.08^{* *}$ \\
$105.40 \pm 10.26^{* *}$ \\
$116.92 \pm 7.91^{* *}$ \\
$137.2 \pm 5.7^{* *, \$}$ \\
$86.3 \pm 4.5^{* *, \$}$ \\
$4.97 \pm 0.93^{*}$ \\
$1.63(1.18-2.30)^{* *}$ \\
$1.03 \pm 0.19^{* *, \$}$ \\
$2.92 \pm 0.68^{*}$ \\
$0.86 \pm 0.30^{*}$ \\
$4.75 \pm 0.89^{* *, \$ \$}$ \\
$24.01(15.96-36.54)^{* *}$ \\
$5.53 \pm 0.38^{* *, \$}$ \\
$6.18(1.26-11.32)^{\star *, \$}$ \\
$5.12(3.13-8.35)^{* *, \$ \$}$ \\
$142.24(93.15-238.83)^{* *, \$ \$}$ \\
$70.00(47.99-110.00)$ \\
$2.80(1.24-4.13)$ \\
$2.54 \pm 1.30^{*}$ \\
$14.44 \pm 5.20^{\$ \$}$
\end{tabular}

\begin{tabular}{c}
\hline $\boldsymbol{P}$ \\
\hline 0.656 \\
0.489 \\
0.000 \\
0.000 \\
0.000 \\
0.000 \\
0.000 \\
0.006 \\
0.000 \\
0.000 \\
0.016 \\
0.035 \\
0.010 \\
0.000 \\
0.000 \\
0.012 \\
0.000 \\
0.000 \\
0.124 \\
0.169 \\
0.032 \\
0.000
\end{tabular}

$257(86.3 \%)$

$118(39.6 \%)$

$263(88.3 \%)$

Data are means \pm s.D. unless indicated otherwise. TG, FINS, hsCRP, HOMA-IR, adipo-IR, HOMA- $\beta$, estradiol, and TT are shown as medians (upper and lower quartiles).

*Significantly different at $P<0.05$ vs control group; **Significantly different at $P<0.01$ vs control group. ${ }^{\$}$ Significantly different at $P<0.05$ vs MHO group;

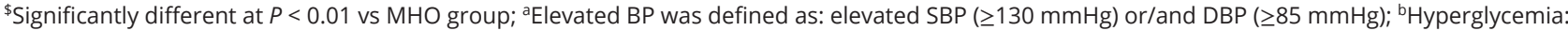
elevated FBG ( $\geq 5.6 \mathrm{mmol} / \mathrm{L}) ;{ }^{c}$ Dyslipidemia: elevated TG $(\geq 1.7 \mathrm{mmol} / \mathrm{L})$ or/and reduced $\mathrm{HDL}-\mathrm{C}(<1.0 \mathrm{mmol} / \mathrm{L}$ for men and $<1.3 \mathrm{mmol} / \mathrm{L}$ for women). Adipo-IR, adipose tissue insulin resistance; DBP, diastolic blood pressure; F, females; FBG, fasting blood glucose; FINS, fasting insulin; HbA1c, hemoglobin A1c; HC, hip circumference; HDL-C, high-density lipoprotein cholesterol; HOMA-IR, homeostasis model assessment of insulin resistance; hsCRP, high-sensitivity C-reactive protein; LDL-C, low-density lipoprotein cholesterol; M, males; MHO, metabolically healthy obesity; MUHO, metabolically unhealthy obesity; NEFA, non-esterified fatty acid; PRL, prolactin;SBP, systolic blood pressure; TC, total cholesterol; TG, triglyceride; TSH, thyroid stimulating hormone; $\mathrm{TT}$, total testosterone; WC, waist circumference.

negatively correlated with WC, TG, NEFA, FBG, HOMA-IR, and adipo-IR, and positively correlated with TSH levels (Table 3). A similar pattern of correlations was also observed when the analysis was stratified by gender (Table 3 ).

Multivariate linear regression analysis was used to further assess the relationship between PRL and potentially relevant variables, including gender, WC, TG, NEFA, FBG, HOMA-IR, adipo-IR, and TSH. The analysis showed that only FBG and adipo-IR levels were negatively associated with PRL levels (FBG: $\beta=-0.263, P<0.05$; adipo-IR: $\beta=$ $-0.464, P<0.01)$.

\section{Multivariate logistic regression analysis to find the independent predictors for MHO}

Multivariable logistic regression analysis was used to identify the potential predictive factors for $\mathrm{MHO}$ and to show that hsCRP and PRL were independent predictors of MHO after adjustment for age, gender, BMI, WC and HC levels (Table 4).

\section{Discussion}

The present study showed that in the obesity group, $15.1 \%$ (53/351) were categorized as MHO. Besides favorable metabolic profiles of blood pressure, glucose, and lipids, the MHO group exhibited significantly higher levels of PRL and lower levels of hsCRP, HOMA-IR, and adipo-IR than the MUHO group. Interestingly, the severe MUHO group showed significantly lower PRL levels than the mild MUHO group but similar PRL levels with the healthy controls. Multivariate linear regression analysis indicated that FBG and adipo-IR were significantly associated with PRL levels, https://ec.bioscientifica.com

https://doi.org/10.1530/EC-21-0040 (c) 2021 The authors Published by Bioscientifica Ltd

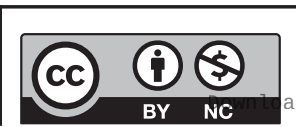

This work is licensed under a Creative Commons Attribution-NonCommercial 4.0 International License. ded from Bioscientifica.com at 04/26/2023 10:04:40AM via free access 


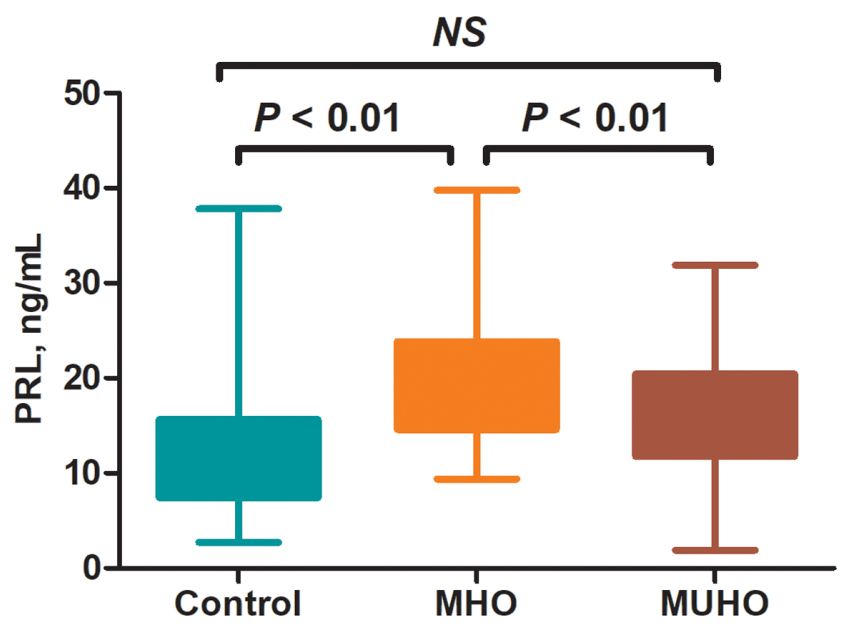

Figure 1

Serum PRL levels of the control, $\mathrm{MHO}$ and $\mathrm{MUHO}$ groups. Values were expressed as a box and whisker with minimum and maximal value. PRL, prolactin; MHO, metabolically healthy obesity; MUHO, metabolically unhealthy obesity.

and multivariable logistic regression analysis showed that hsCRP and PRL were independent predictors of MHO.

The present study showed that $15.1 \%$ were categorized as $\mathrm{MHO}$ in the obesity group, which was similar with many previous studies $(5,6,7)$. Despite comparable BMI levels, relatively lower levels of HOMA-IR and adipo-IR were observed in the MHO group, as compared to the MUHO group. In addition, our study found that the MHO group had significantly lower hsCRP levels than the MUHO group $(24,25)$. A chronic inflammatory state has been demonstrated to cause insulin resistance via activating intracellular inflammatory pathways in many previous studies $(26,27)$. Therefore, the relatively lower inflammatory state and elevated insulin sensitivity in both systemic and adipose tissue seem to be responsible for favorable metabolic parameters in the MHO phenotype.

Accumulating evidence has suggested that PRL as a metabolic hormone to regulate energy metabolism $(8,9$, $10,11,12,13,14,15,16)$. The present study showed that the MHO patients had significantly higher circulating PRL levels than the control and MUHO group. Correlation analyses found that the circulating PRL was negatively correlated with FBG, HOMA-IR, and adipo-IR in all participants. And multivariate linear regression analysis indicated that levels of FBG and adipo-IR were significantly associated with PRL levels. Adipocytes of rodents and humans expressed PRL receptors $(10,28)$. Previous studies found that increased PRL levels were involved in healthy expansion of adipose tissue in both rodents and humans, especially during obesity $(10,29)$. In diet-induced obese rats, PRL administration inhibited adipocyte hypertrophy,
Table 2 Clinical characteristics of the mild and severe MUHO groups.

\begin{tabular}{|c|c|c|c|}
\hline Parameters & $\begin{array}{l}\text { Mild MUHO group } \\
\qquad(n=151)\end{array}$ & $\begin{array}{l}\text { Severe MUHO group } \\
(n=147)\end{array}$ & $\boldsymbol{P}$ \\
\hline Age, years & $31.2 \pm 7.6$ & $31.9 \pm 7.1$ & 0.399 \\
\hline Gender, M/F, $n$ & $46 / 105$ & $55 / 92$ & 0.384 \\
\hline $\mathrm{BMI}, \mathrm{kg} / \mathrm{m}^{2}$ & $33.96 \pm 4.41$ & $34.05 \pm 4.29$ & 0.110 \\
\hline WC, $\mathrm{cm}$ & $103.58 \pm 13.30$ & $107.32 \pm 13.47$ & 0.055 \\
\hline $\mathrm{HC}, \mathrm{cm}$ & $114.29 \pm 12.16$ & $118.24 \pm 9.70$ & 0.060 \\
\hline $\mathrm{SBP}, \mathrm{mmHg}$ & $132.8 \pm 5.5$ & $143.5 \pm 6.2$ & 0.000 \\
\hline $\mathrm{DBP}, \mathrm{mmHg}$ & $85.6 \pm 4.9$ & $87.9 \pm 4.4$ & 0.000 \\
\hline $\mathrm{TC}, \mathrm{mmol} / \mathrm{L}$ & $4.94 \pm 0.69$ & $5.02 \pm 0.84$ & 0.105 \\
\hline $\mathrm{TG}, \mathrm{mmol} / \mathrm{L}$ & $1.27(0.98-1.62)$ & $2.14(1.70-2.96)$ & 0.000 \\
\hline $\begin{array}{l}\mathrm{HDL}-\mathrm{C} \\
\mathrm{mmol} / \mathrm{L}\end{array}$ & $1.08 \pm 0.19$ & $0.97 \pm 0.18$ & 0.000 \\
\hline $\begin{array}{l}\text { LDL-C, } \\
\text { mmol/L }\end{array}$ & $2.89 \pm 0.70$ & $2.95 \pm 0.61$ & 0.250 \\
\hline NEFA, mmol/L & $0.77 \pm 0.21$ & $0.90 \pm 0.34$ & 0.031 \\
\hline FBG, mmol/L & $4.57 \pm 0.78$ & $5.09 \pm 0.94$ & 0.000 \\
\hline FINS, mIU/L & $19.52(13.10-31.06)$ & 27.31 (19.44-41.16) & 0.021 \\
\hline $\mathrm{HbA} 1 \mathrm{c}, \%$ & $5.49 \pm 0.41$ & $5.56 \pm 0.36$ & 0.180 \\
\hline hsCRP, mg/L & $5.54(0.94-12.25)$ & 6.95 (1.84-9.76) & 0.036 \\
\hline HOMA-IR & $4.87(3.02-6.50)$ & $6.96(4.22-10.15)$ & 0.000 \\
\hline adipo-IR & 125.53 (68.07-149.69) & $156.88(121.66-273.93)$ & 0.013 \\
\hline $\begin{array}{l}\text { Estradiol, } \\
\mathrm{pg} / \mathrm{mL}\end{array}$ & $72.50(49.84-115.00)$ & $67.00(47.86-104.10)$ & 0.548 \\
\hline $\mathrm{TT}, \mathrm{ng} / \mathrm{mL}$ & $2.75(1.22-3.86)$ & $2.98(1.27-4.92)$ & 0.332 \\
\hline $\mathrm{TSH}, \mu \mathrm{IU} / \mathrm{mL}$ & $2.50 \pm 1.37$ & $2.60 \pm 1.22$ & 0.578 \\
\hline $\mathrm{PRL}, \mathrm{ng} / \mathrm{mL}$ & $15.95 \pm 5.40$ & $12.04 \pm 4.46$ & 0.004 \\
\hline
\end{tabular}

Data are means \pm S.D. unless indicated otherwise. TG, FINS, hsCRP, HOMA-IR, adipo-IR, HOMA- $\beta$, estradiol, and TT are shown as medians (upper and lower quartiles).

Adipo-IR, adipose tissue insulin resistance; DBP, diastolic blood pressure; F, females; FBG, fasting blood glucose; FINS, fasting insulin; HbA1C, hemoglobin A1 c; HC, hip circumference; HDL-C, high-density lipoprotein cholesterol; HOMA-IR, homeostasis model assessment of insulin resistance; hsCRP, high-sensitivity C-reactive protein; LDL-C, low-density lipoprotein cholesterol; M, males; MHO, metabolically healthy obesity; MUHO, metabolically unhealthy obesity; NEFA, non-esterified fatty acid; PRL, prolactin; SBP, systolic blood pressure; TC, total cholesterol; TG, triglyceride; $\mathrm{TSH}$, thyroid stimulating hormone; TT, total testosterone; WC, waist circumference.

down-regulated inflammatory cytokine expression in visceral adipose tissue, and alleviated insulin resistance (10, 30). PRL receptor knockout mice exhibited higher insulin resistance and severe adipose tissue dysfunction (10). Therefore, increased circulating PRL might be a compensatory response for favoring energy metabolism during obesity. Consistently, the concept of homeostasis functionally increased transient hyperprolactinemia (HomeoFIT-PRL) which has been proposed recently by Macotela et al. and this proposed definition reveals that elevated PRL levels favor metabolic homeostasis in a situation of metabolic need or challenge (20).

Several cohort studies have demonstrated that individuals with a lower circulating PRL level have an increased risk of insulin resistance, diabetes, and metabolic syndrome $(13,14,15)$. In the present study,

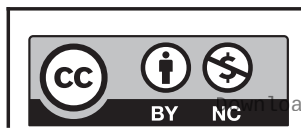

This work is licensed under a Creative Commons Attribution-NonCommercial 4.0 International License. ded from Bioscientifica.com at 04/26/2023 10:04:40AM 
Table 3 Correlation between PRL and clinical parameters in all participants.

\begin{tabular}{l}
\hline Parameters \\
\hline Age \\
BMI \\
WC \\
HC \\
SBP \\
DBP \\
TC \\
TG \\
HDL-C \\
LDL-C \\
NEFA \\
FBG \\
FINS \\
HbA1C \\
hSCRP \\
HOMA-IR \\
adipo-IR \\
Estradiol \\
TT \\
TSH
\end{tabular}

\begin{tabular}{ccc}
\hline \multicolumn{3}{c}{ PRL (all) } \\
\hline \multicolumn{1}{r}{$r$} & $P$ \\
\cline { 1 - 1 }-0.006 & & 0.930 \\
-0.141 & & 0.357 \\
-0.137 & & 0.032 \\
-0.172 & & 0.324 \\
-0.086 & & 0.764 \\
-0.076 & & 0.221 \\
-0.260 & & 0.000 \\
0.022 & & 0.726 \\
-0.093 & & 0.135 \\
-0.222 & & 0.002 \\
-0.136 & & 0.030 \\
-0.080 & & 0.225 \\
-0.010 & & 0.879 \\
-0.012 & & 0.963 \\
-0.244 & & 0.000 \\
-0.302 & 0.000 \\
-0.007 & 0.905 \\
0.106 & 0.065 \\
0.143 & 0.037
\end{tabular}

\begin{tabular}{rrr}
\hline \multicolumn{2}{c}{ PRL (male) } \\
\hline \multicolumn{1}{c}{$r$} & & $P$ \\
\cline { 1 - 1 } 0.025 & & 0.098 \\
-0.151 & & 0.762 \\
-0.071 & & 0.023 \\
-0.194 & & 0.431 \\
-0.077 & & 0.874 \\
0.113 & & 0.167 \\
-0.162 & & 0.008 \\
0.031 & & 0.617 \\
-0.037 & & 0.236 \\
-0.601 & & 0.000 \\
-0.132 & & 0.043 \\
-0.036 & & 0.842 \\
-0.112 & & 0.172 \\
-0.003 & & 0.982 \\
-0.228 & & 0.000 \\
-0.324 & & 0.000 \\
0.079 & 0.497 \\
0.226 & 0.198 \\
0.174 & 0.033
\end{tabular}

\begin{tabular}{|c|c|}
\hline \multicolumn{2}{|c|}{ PRL (female) } \\
\hline$r$ & $P$ \\
\hline-0.123 & 0.056 \\
\hline-0.076 & 0.465 \\
\hline-0.138 & 0.045 \\
\hline-0.173 & 0.319 \\
\hline-0.161 & 0.502 \\
\hline-0.090 & 0.773 \\
\hline-0.114 & 0.077 \\
\hline-0.246 & 0.000 \\
\hline 0.029 & 0.769 \\
\hline-0.095 & 0.139 \\
\hline-0.290 & 0.012 \\
\hline-0.155 & 0.033 \\
\hline-0.120 & 0.134 \\
\hline 0.056 & 0.400 \\
\hline-0.142 & 0.531 \\
\hline-0.232 & 0.000 \\
\hline-0.296 & 0.000 \\
\hline-0.093 & 0.181 \\
\hline-0.104 & 0.187 \\
\hline 0.240 & 0.000 \\
\hline
\end{tabular}

Adipo-IR, adipose tissue insulin resistance; DBP, diastolic blood pressure; FBG, fasting blood glucose; FINS, fasting insulin; HbA1c, hemoglobin A1c; HC, hip circumference; HDL-C, high-density lipoprotein cholesterol; HOMA-IR, homeostasis model assessment of insulin resistance; hsCRP, high-sensitivity C-reactive protein; LDL-C, low-density lipoprotein cholesterol; NEFA, non-esterified fatty acid; PRL, prolactin; SBP, systolic blood pressure; TC, total cholesterol; TG, triglyceride; TSH, thyroid stimulating hormone; TT, total testosterone; WC, waist circumference.

the circulating PRL level of the MUHO group was similar with the healthy controls, but significantly lower than that of $\mathrm{MHO}$ group. Interestingly, the severe MUHO group showed significantly lower PRL levels than the mild MUHO group, but similar PRL levels with the healthy controls. These results might suggest that the compensatory increased PRL did not occur as expected in MUHO patients, especially in severe MUHO patients. In order to investigate the association between PRL and metabolic alterations in the early stage of metabolic abnormalities, participants with a history of diabetes or taking any medications which influence glucose, lipid, or blood pressure were excluded from the present study. Therefore, the metabolic abnormality was relatively mild in the enrolled MUHO patients, which might explain why reduced PRL levels were not observed in MUHO subjects.

Levels of BP, glucose, and atherogenic lipid were relatively higher in the $\mathrm{MHO}$ group than the healthy control group, although they remained within the normal range. Furthermore, significantly increased hsCRP, HOMA-IR, and adipo-IR levels were also observed in the MHO group when compared with healthy controls. These results suggested that MHO manifested elevated inflammatory factor expression, decreased insulin sensitivity, and compensatory increased insulin secretion, despite without overt metabolic abnormality. Moreover, many studies have confirmed that MHO subjects were more prone to develop multiple cardiovascular diseases over time

Table 4 Multivariate logistic regression analysis to find the independent predictors for MHO.

\begin{tabular}{|c|c|c|}
\hline \multirow[b]{2}{*}{ Parameters } & \multicolumn{2}{|c|}{ MHO } \\
\hline & $P$ & OR $(95 \% \mathrm{Cl})$ \\
\hline Age & 0.382 & $0.941(0.773-1.120)$ \\
\hline Male gender & 0.494 & $0.902(0.695-1.248)$ \\
\hline $\mathrm{BMI}$ & 0.079 & $0.892(0.593-1.011)$ \\
\hline WC & 0.347 & 0.899 (0.639-1.053) \\
\hline $\mathrm{HC}$ & 0.690 & $0.938(0.703-1.141)$ \\
\hline HsCRP & 0.002 & $0.824(0.717-0.943)$ \\
\hline PRL & 0.001 & $1.211(1.017-1.464)$ \\
\hline
\end{tabular}

HC, hip circumference; hsCRP, high-sensitivity C-reactive protein; MHO, metabolically healthy obesity; OR, odds ratio; PRL, prolactin; WC, waist circumference.

https://ec.bioscientifica.com

https://doi.org/10.1530/EC-21-0040 (c) 2021 The authors Published by Bioscientifica Ltd

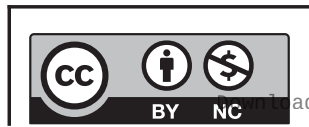

This work is licensed under a Creative Commons Attribution-NonCommercial 4.0 International License. ded from Bioscientifica.com at 04/26/2023 10:04:40AM via free access 
as compared with normal-weight healthy individuals, even in the absence of metabolic abnormalities (31). Therefore, every obese individual should be encouraged to achieve normal weight in the long term.

The present study has some limitations. First, a single blood sample might not well reveal daily PRL secretion, on account of the pulsatile release of PRL (32). Although mostly pulsatile during the night, PRL secretion is relatively constant in the daytime (32). Thus, for the sake of narrowing PRL variation as much as possible, blood samples were taken between 8:00 and 9:00 $\mathrm{h}$ in the present study. Secondly, this study is a single center study with a relatively small sample size, which may have introduced selective bias and limited the generalizability of findings. Thirdly, PRL levels vary during the menstrual cycle but the present study did not standardize for the menstrual cycle (19). Finally, in fact, a universally accepted criterion for MHO has not yet been established so far (6). The present study used a strict definition of $\mathrm{MHO}$ proposed by Ortega, which was accepted by most previous studies $(7,23,33$, 34). Further research is needed to better understand the role of PRL and other relevant parameters in different obesity phenotypes and to elucidate the precise mechanisms underlying the transition from MHO to MUHO.

In conclusion, the MHO group had significantly increased circulating PRL levels when compared with the control and MUHO groups, and multivariable logistic regression analysis showed that PRL were independently associated with MHO. Our findings suggested that increased circulating PRL might be a compensatory response for favoring energy metabolism during obesity. However, further studies, including human, animal, and cell experiments, are still needed to confirm these results and get better insight into the underlying mechanisms.

\section{Declaration of interest}

The authors declare that there is no conflict of interest to that could be perceived as prejudicing the impartiality of the research reported.

\section{Funding}

This work was supported by grants from the Beijing Talents foundation (2018-12) to J L and the Chinese National Natural Science Foundation (No. 81770792) and Key Projects of Science and Technology Planning of Beijing Municipal Education Commission (KZ201810025038) to G W. The funders had no role in study design, data collection and analysis, decision to publish, or preparation of the manuscript.

\section{Author contribution statement}

G W was involved in final approval of the manuscript, conception or design; J L, L Z, J F, and $Q$ W were involved in acquisition, analysis, or interpretation of data; $\mathrm{J} L$ and $\mathrm{L} Z \mathrm{Z}$ were involved in drafting the work or revising.

\section{References}

1 Flegal KM, Kit BK, Orpana H \& Graubard BI. Association of all-cause mortality with overweight and obesity using standard body mass index categories: a systematic review and meta-analysis. Journal of American Medical Association 2013309 71-82. (https://doi. org/10.1001/jama.2012.113905)

2 Jensen MD, Ryan DH, Apovian CM, Ard JD, Comuzzie AG, Donato KA, Hu FB, Hubbard VS, Jakicic JM, Kushner RF, et al. 2013 AHA/ACC/TOS guideline for the management of overweight and obesity in adults: a report of the American College of Cardiology/ American Heart Association Task Force on Practice Guidelines and the Obesity Society. Journal of the American College of Cardiology 2014 63 2985-3023. (https://doi.org/10.1016/j.jacc.2013.11.004)

3 Emerging Risk Factors Collaboration, Wormser D, Kaptoge S, Di Angelantonio E, Wood AM, Pennells L, Thompson A, Sarwar N, Kizer JR, Lawlor DA, et al. Separate and combined associations of body-mass index and abdominal adiposity with cardiovascular disease: collaborative analysis of 58 prospective studies. Lancet 2011 377 1085-1095. (doi:10.1016/S0140-6736(11)60105-0)

4 Schulze MB. Metabolic health in normal-weight and obese individuals. Diabetologia 201962 558-566. (https://doi.org/10.1007/ s00125-018-4787-8)

5 Stefan N, Haring HU, Hu FB \& Schulze MB. Metabolically healthy obesity: epidemiology, mechanisms, and clinical implications. Lancet: Diabetes and Endocrinology 20131 152-162. (doi:10.1016/ S2213-8587(13)70062-7)

6 Tsatsoulis A \& Paschou SA. Metabolically healthy obesity: criteria epidemiology, controversies, and consequences. Current Obesity Reports 20209 109-120. (https://doi.org/10.1007/s13679-020-00375-0)

7 Bluher M. Metabolically healthy obesity. Endocrine Reviews 202041 405-420. (https://doi.org/10.1210/endrev/bnaa004)

8 Bernard V, Young J \& Binart N. Prolactin - a pleiotropic factor in health and disease. Nature Reviews: Endocrinology 201915 356-365. (https://doi.org/10.1038/s41574-019-0194-6)

9 Ben-Jonathan N \& Hugo E. Prolactin (PRL) in adipose tissue: regulation and functions. Advances in Experimental Medicine and Biology 2015846 1-35. (https://doi.org/10.1007/978-3-319-121147_1)

10 Ruiz-Herrera X, de Los Rios EA, Diaz JM, Lerma-Alvarado RM, Martinez de la Escalera L, Lopez-Barrera F, Lemini M, Arnold E, Martinez de la Escalera G, Clapp C, et al. Prolactin promotes adipose tissue fitness and insulin sensitivity in obese males. Endocrinology 2017158 56-68. (https://doi.org/10.1210/en.2016-1444)

11 Holstad M \& Sandler S. Prolactin protects against diabetes induced by multiple low doses of streptozotocin in mice. Journal of Endocrinology 1999163 229-234. (https://doi.org/10.1677/joe.0.1630229)

12 Zhang P, Ge Z, Wang H, Feng W, Sun X, Chu X, Jiang C, Wang Y, Zhu D \& Bi Y. Prolactin improves hepatic steatosis via CD36 pathway. Journal of Hepatology 201868 1247-1255. (https://doi. org/10.1016/j.jhep.2018.01.035)

13 Li J, Rice MS, Huang T, Hankinson SE, Clevenger CV, Hu FB \& Tworoger SS. Circulating prolactin concentrations and risk of type 2 diabetes in US women. Diabetologia 201861 2549-2560. (https://doi. org/10.1007/s00125-018-4733-9)

14 Corona G, Wu FC, Rastrelli G, Lee DM, Forti G, O'Connor DB, O'Neill TW, Pendleton N, Bartfai G, Boonen S, et al. Low prolactin is associated with sexual dysfunction and psychological or metabolic disturbances in middle-aged and elderly men: the European Male Aging Study (EMAS). Journal of Sexual Medicine 201411 240-253. (https://doi.org/10.1111/jsm.12327)

15 Chirico V, Cannavo S, Lacquaniti A, Salpietro V, Mandolfino M, Romeo PD, Cotta O, Munafo C, Giorgianni G, Salpietro C, et al. Prolactin in obese children: a bridge between inflammation and metabolic-endocrine dysfunction. Clinical Endocrinology 201379 537-544. (https://doi.org/10.1111/cen.12183)

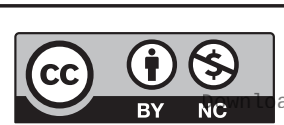


16 Copinschi G, De Laet MH, Brion JP, Leclercq R, L'Hermite M, Robyn C, Virasoro E \& Van Cauter E. Simultaneous study of cortisol, growth hormone and prolactin nyctohemeral variations in normal and obese subjects. Influence of prolonged fasting in obesity. Clinical Endocrinology 19789 15-26. (https://doi. org/10.1111/j.1365-2265.1978.tb03568.x)

17 Kok P, Roelfsema F, Frolich M, Meinders AE \& Pijl H. Prolactin release is enhanced in proportion to excess visceral fat in obese women. Journal of Clinical Endocrinology and Metabolism 200489 4445-4449. (https://doi.org/10.1210/jc.2003-032184)

18 Camhi SM, Must A, Gona PN, Hankinson A, Odegaard A, Reis J, Gunderson EP, Jacobs DR \& Carnethon MR. Duration and stability of metabolically healthy obesity over 30 years. International Journal of Obesity 201943 1803-1810. (https://doi.org/10.1038/ s41366-018-0197-8)

19 Melmed S, Casanueva FF, Hoffman AR, Kleinberg DL, Montori VM, Schlechte JA, Wass JA \& Endocrine Society. Diagnosis and treatment of hyperprolactinemia: an Endocrine Society clinical practice guideline. Journal of Clinical Endocrinology and Metabolism 201196 273-288. (https://doi.org/10.1210/jc.2010-1692)

20 Macotela Y, Triebel J \& Clapp C. Time for a new perspective on prolactin in metabolism. Trends in Endocrinology and Metabolism 2020 31 276-286. (https://doi.org/10.1016/j.tem.2020.01.004)

21 Matthews DR, Hosker JP, Rudenski AS, Naylor BA, Treacher DF \& Turner RC. Homeostasis model assessment: insulin resistance and beta-cell function from fasting plasma glucose and insulin concentrations in man. Diabetologia 198528 412-419. (https://doi. org/10.1007/BF00280883)

22 Gastaldelli A, Gaggini M \& DeFronzo RA. Role of adipose tissue insulin resistance in the natural history of type 2 diabetes: results from the San Antonio metabolism study. Diabetes 201766 815-822. (https://doi.org/10.2337/db16-1167)

23 Ortega FB, Lavie CJ \& Blair SN. Obesity and cardiovascular disease. Circulation Research 2016118 1752-1770. (https://doi.org/10.1161/ CIRCRESAHA.115.306883)

24 Karelis AD \& Rabasa-Lhoret R. Obesity: can inflammatory status define metabolic health? Nature Reviews: Endocrinology 20139 694-695. (https://doi.org/10.1038/nrendo.2013.198)

25 van Beek L, Lips MA, Visser A, Pijl H, Ioan-Facsinay A, Toes R, Berends FJ, Willems van Dijk K, Koning F \& van Harmelen V. Increased systemic and adipose tissue inflammation differentiates obese women with T2DM from obese women with normal glucose tolerance. Metabolism: Clinical and Experimental 201463 492-501. (https://doi.org/10.1016/j.metabol.2013.12.002)

26 Xu H, Barnes GT, Yang Q, Tan G, Yang D, Chou CJ, Sole J, Nichols A, Ross JS, Tartaglia LA, et al. Chronic inflammation in fat plays a crucial role in the development of obesity-related insulin resistance. Journal of Clinical Investigation 2003112 1821-1830. (https://doi. org/10.1172/JCI19451)

27 Lumeng CN, Bodzin JL \& Saltiel AR. Obesity induces a phenotypic switch in adipose tissue macrophage polarization. Journal of Clinical Investigation 2007117 175-184. (https://doi.org/10.1172/JCI29881)

28 Ling C, Svensson L, Oden B, Weijdegard B, Eden B, Eden S \& Billig H. Identification of functional prolactin (PRL) receptor gene expression: PRL inhibits lipoprotein lipase activity in human white adipose tissue. Journal of Clinical Endocrinology and Metabolism $2003 \mathbf{8 8}$ 1804-1808. (https://doi.org/10.1210/jc.2002-021137)

29 Ponce AJ, Galvan-Salas T, Lerma-Alvarado RM, Ruiz-Herrera X, Hernandez-Cortes T, Valencia-Jimenez R, Cardenas-Rodriguez LE, Martinez de la Escalera G, Clapp C \& Macotela Y. Low prolactin levels are associated with visceral adipocyte hypertrophy and insulin resistance in humans. Endocrine 202067 331-343. (https://doi. org/10.1007/s12020-019-02170-x)

30 Carre N \& Binart N. Prolactin and adipose tissue. Biochimie 201497 16-21. (https://doi.org/10.1016/j.biochi.2013.09.023)

31 Hansen L, Netterstrom MK, Johansen NB, Ronn PF, Vistisen D, Husemoen LLN, Jorgensen ME, Rod NH \& Faerch K. Metabolically healthy obesity and ischemic heart disease: a 10-year follow-up of the Inter99 study. Journal of Clinical Endocrinology and Metabolism 2017102 1934-1942. (https://doi.org/10.1210/jc.2016-3346)

32 Partsch CJ, Lerchl A \& Sippell WG. Characteristics of pulsatile and circadian prolactin release and its variability in men. Experimental and Clinical Endocrinology and Diabetes 1995103 33-43. (https://doi. org/10.1055/s-0029-1211327)

33 Romagnolli C, Bensenor IM, Santos IS, Lotufo PA \& Bittencourt MS Impact of metabolically healthy obesity on carotid intima-media thickness - the Brazilian Longitudinal Study of Adult Health. Nutrition, Metabolism, and Cardiovascular Diseases 202030 915-921. (https://doi.org/10.1016/j.numecd.2020.02.012)

34 Catoi AF \& Busetto L. Metabolically healthy obesity and bariatric surgery. Obesity Surgery 201929 2989-3000. (https://doi.org/10.1007/ s11695-019-03964-8)

Received in final form 16 March 2021

Accepted 31 March 2021

Accepted Manuscript published online 31 March 2021 https://ec.bioscientifica.com

https://doi.org/10.1530/EC-21-0040 (c) 2021 The authors Published by Bioscientifica Ltd
This work is licensed under a Creative Commons Attribution-NonCommercial 4.0 International License. ded from Bioscientifica.com at 04/26/2023 10:04:40AM 